EOMmUn: Communication et organisation

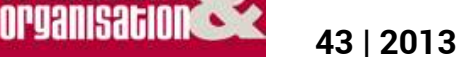

Réseaux sociaux entre médias et médiations

\title{
Vers un modèle d'efficience des collectifs
}

\author{
Pierre-Michel Riccio
}

\section{OpenEdition}

Journals

Édition électronique

URL : http://journals.openedition.org/communicationorganisation/4107

DOI : 10.4000/communicationorganisation.4107

ISSN : 1775-3546

\section{Éditeur}

Presses universitaires de Bordeaux

\section{Édition imprimée}

Date de publication : 1 juin 2013

Pagination : $37-46$

ISBN : 978-2-86781-883-7

ISSN : 1168-5549

\section{Référence électronique}

Pierre-Michel Riccio, "Vers un modèle d'efficience des collectifs », Communication et organisation [En ligne], 43 | 2013, mis en ligne le 01 juin 2015, consulté le 20 avril 2019. URL : http://

journals.openedition.org/communicationorganisation/4107; DOI : 10.4000/ communicationorganisation.4107 


\title{
Vers un modèle d'efficience des collectifs
}

\author{
Pierre-Michel Riccio'
}

L'objectif de cet article est de jeter les premières bases d'un modèle d'efficience des collectifs, qui, sans viser l'identification d'une "solution idéale " assez utopique compte tenu de la complexité du monde et de son évolution permanente, permettrait d'améliorer de façon progressive la performance d'un collectif dans l'action en relation avec les moyens qui sont disponibles ou mobilisables ${ }^{2}$.

Le développement des réseaux numériques - internet et intranet - et l'internationalisation de la concurrence font que le partage de connaissances est devenu un enjeu majeur de compétitivité pour les entreprises comme pour les organisations.

Aussi, le point de départ de ce travail de recherche, a été d'imaginer de nouveaux dispositifs de partage des connaissances fondés sur les Technologies de l'Information et de la Communication (TIC) pour faciliter le travail collaboratif.

Cette tâche est généralement confiée à des équipes de spécialistes en Sciences et Technologies de l'Information et de la Communication (STIC) qui maîtrisent la dimension technique des outils virtuels. Ils utilisent pour ce faire des approches comme le langage unifié de modélisation UML (Booch et al., 2005) qui propose une large palette de modèles pour appréhender le réel et le traduire in fine en code logiciel exécutable par des ordinateurs. Ces modèles concernent les objets à manipuler, les activités et leur enchaînement, la mise en séquence des actions, ou encore le déroulement des scénarios d'usage. L'objectif est d'appréhender la situation dans ses différentes dimensions pour créer l'outil « idéal».

1 Pierre-Michel Riccio est ingénieur du Conservatoire National des Arts \& Métiers, diplômé en management et docteur en sciences de l'information et de la communication de l'université de Montpellier, habilité à diriger des recherches de l'université d'Aix-Marseille. Après dix années en entreprises puis vingt années en laboratoires, il est aujourd'hui enseignant-chercheur / responsable opérationnel du centre de recherche LGI2P de l'Ecole des mines d'Alès à Nîmes ; Pierre-Michel.Riccio@mines-ales.fr

2 Nous tenons à remercier le Pr. Serge Agostinelli (Université Aix-Marseille) et le Pr. Jacky Montmain (Ecole des mines d'Alès) dont les travaux, en SHS et en STIC, nous ont largement inspirés dans la mise en place de cette démarche. 


\section{Cette démarche peut-elle / doit-elle être centrée sur l'outil ?}

Michel Foucault a montré que le dispositif est : « un ensemble résolument hétérogène, comportant des discours, des institutions, des aménagements architecturaux, des décisions réglementaires, des lois, des mesures administratives, des énoncés scientifiques, des propositions philosophiques, morales, philanthropiques, bref : du dit, aussi bien que du non-dit » (Foucault, 1977 : 299).

Pour Michel Foucault, le dispositif se matérialise par le réseau établi entre ces différents éléments. Il possède une fonction stratégique dominante. Il est naturellement inscrit dans un jeu de pouvoir. Mais, il est aussi lié à une ou des bornes de savoir qui en naissent et le conditionnent.

Aussi, pour imaginer et mettre en œuvre un système de partage des connaissances, partie intégrante d'un dispositif, une approche STIC ne suffit pas. Il est nécessaire d'élargir le cadre pour comprendre la situation en s'appuyant sur des modèles propres aux Sciences Humaines et Sociales (SHS) avec pour objectif d'appréhender les différents contextes : de construction, d'aménagement, d'utilisation.

Il existe de nombreux modèles dans le domaine des SHS qui peuvent être mis à contribution pour appréhender la création ou l'aménagement de dispositifs en situation. Nous nous limiterons ici aux approches que nous avons utilisées dans le cadre de nos travaux en sciences de l'information et de la communication (SIC) (Riccio, 2003, 2010) :

La théorie sémio-contextuelle des communications (Mucchielli, 2000) permet d'appréhender les processus de communication qui impactent les contextes de la situation (en particulier enjeux et normes). Elle facilite la compréhension des interrelations entre par exemple discours, institutions, et aménagements architecturaux (comme peuvent l'être des systèmes technologiques) qui sont parties d'un dispositif.

La théorie systémique des communications (Mucchielli, 1999) a pour objet de faciliter l'identification des causalités circulaires (en particulier boucles d'interaction et paradoxes) pour éclairer la compréhension de la situation. Elle facilite le cadrage de l'étude et le repérage des anomalies ou dysfonctionnements entre composantes d'un dispositif.

L'approche sémiotique, mise en œuvre dans nos travaux du point de vue de Peirce (Marty, 1992), permet quand à elle d'appréhender la situation à travers l'étude et l'analyse des signes (du qualisigne à l'argument). Cette démarche basée sur un triptyque (objet, signe, interprétant) et la décomposition des phénomènes en niveaux complémentaires) peut faciliter l'appréhension de certaines dimensions d'un dispositif du point de vue des acteurs dans l'action.

Dans le même esprit, la sociologie de la traduction (Akrich, 2006) permet, à travers l'analyse des asymétries entre acteurs individuels et collectifs, d'éclairer la position des forces en présence et dans une approche diachronique leur poids ou impact dans la création ou l'aménagement d'un dispositif. 
Mais, quelle est la compatibilité effective ou à construire entre les modèles STIC et SHS ? Quelles sont les dimensions sous jacentes, qui compte tenu de la spécificité de l'objectif, contribuent à un meilleur partage des connaissances ? Quelles sont celles qui peuvent, en facilitant la montée en niveaux d'abstraction, être mises à contribution?

\section{En partant des modèles STIC}

Dans le domaine des STIC il existe de nombreuses méthodes, généralement dotées d'un formalisme graphique, pour construire un modèle de la situation et le traduire ensuite en code exécutable par des calculateurs.

Depuis quelques années, de nouveaux langages se généralisent comme le $\mathrm{C}++$ et Java. Ces langages dits « objets » sont accompagnés par l'émergence de nouveaux modèles " orientés objets ». Après une période de tâtonnements assez longue, plusieurs méthodes, qui permettent d'atteindre le même objectif en empruntant différents chemins, ont émergé. Ce sont celles de : Grady Booch, Jim Rumbaugh et Ivar Jacobson. En 1995, ces auteurs ont présenté une méthode unifiée, qui deviendra au début de l'année 1997 la première version du langage unifié de modélisation UML, mise rapidement à la disposition de tous sur les réseaux numériques et depuis régulièrement mise à jour (Booch et al., 2005).

UML est aujourd'hui l'environnement de référence au niveau international pour la construction de modèles de systèmes techniques ou technologiques. Son succès est certainement lié : à sa standardisation, au fort soutien des utilisateurs, à sa mise à jour régulière (de nombreuses manifestations scientifiques lui sont dédiées), au large choix de possibilités proposées (environ une dizaine), et au grand nombre d'outils qui facilitent pour les concepteurs les tâches de modélisation.

UML propose neuf types de diagrammes qui constituent autant de points de vues sur un système en construction : 1) le diagramme de classes, 2) les cas d'utilisation, 3) les diagrammes d'objets, 4) les diagrammes de séquences, 5) les diagrammes d'états-transitions, 6) les diagrammes de séquences, 7) les diagrammes d'activités, 8) les diagrammes de composants, et 9) les diagrammes de déploiement.

Pour illustrer la démarche, voici une description rapide de trois de ces « modèles ":

Le diagramme de classes représente la vue statique d'un système. Il est composé de classes qui décrivent des familles d'objets possédant la même structure et le même comportement (Stefik and Bobrow, 1986) et de relations qui sont pour l'essentiel au nombre de quatre (association, agrégation, composition et héritage). La richesse de la démarche est assurée par ces différents types de relations : l'association matérialise un lien entre deux classes d'objets de même niveau (par exemple des personnes utilisent des voitures), la composition matérialise un lien d'inclusion (par exemple une 
voiture est composée d'une carrosserie, d'un moteur et d'une transmission), et l'héritage matérialise un lien de dérivation (par exemple la voiture de sport est une voiture qui possède en sus certaines caractéristiques au niveau du moteur, du volant, des suspensions ou des roues).

Les cas d'utilisation connus aussi sous la dénomination anglaise de "uses cases " (Jacobson, 1992) ont pour objet de faciliter la description sous forme graphique ou textuelle de l'utilisation d'un système. Ils sont organisés autour de trois composantes : les acteurs, les cas d'utilisations, et le système. L'acteur représente le rôle joué par une personne ou un élément en interaction avec le système. Les cas d'utilisations sont déterminés par analyse des séquences d'interactions entre les acteurs et le système. Ils font l'objet d'une représentation graphique, mais aussi d'une description textuelle qui précise : le début et la fin du cas d'utilisation, les interactions, les informations échangées, ainsi que la chronologie et l'origine des informations. Le « système » est construit progressivement à partir de la juxtaposition de l'ensemble des cas d'utilisations.

Le diagramme d'activités est une variante du diagramme états-transitions fondé sur l'identification de périodes de stabilité relative d'un système appelée états et sur l'émergence d'évènements qui après une période de transition conduisent à un changement d'état. Ces diagrammes servent à construire de façon rigoureuse puis visualiser l'enchaînement des opérations. Les activités sont représentées sous la forme de rectangles dotés de demi-cercles à leurs extrémités. La transition est représentée par une flèche. Ce type de diagramme possède plusieurs variantes.

\section{La construction de modèles sur le terrain}

Au début des années 2000, les dispositifs que nous avons imaginés (le terme dispositif est entendu ici dans le sens que lui attribuions à l'époque : c'est-à-dire comme des systèmes techniques / technologiques facilitant naturellement la médiation entre les acteurs) avaient pour vocation de faciliter l'échange entre scientifiques (Riccio, 2010). Ils étaient articulés autour de plateformes numériques d'échange, avec pour ambition de renforcer l'efficacité du collectif dans l'action, de créer une véritable « intelligence collective »(Penalva, 2006).

Pour construire ces plateformes ou dispositifs, fondés sur des systèmes de gestion de contenus (Content Management Systems ou CMS), nous nous sommes appuyés sur des modèles STIC issus d'UML. Les diagrammes d'activités et les cas d'utilisation nous ont permis de collecter les «besoins " exprimés par les commanditaires et utilisateurs, pour traduire ensuite ces besoins en « objets » (éléments de base des nouveaux langages de programmation des calculateurs) via les diagrammes de classes.

Nous avons assez rapidement constaté que si les systèmes techniques construits en nous appuyant sur les STIC étaient pleinement opérationnels, le dispositif (entendu dans le sens proposé par Michel Foucault) fonctionnait de façon peu satisfaisante. Les échanges entre scientifiques étaient très limités 
et les plateformes numériques d'échange utilisées de facto essentiellement par les cellules de direction de programmes.

Actant cette faiblesse, nous avons alors lancé plusieurs études en SHS afin de comprendre pourquoi le dispositif ne fonctionnait pas comme nous l'avions imaginé.

L'approche sémio-contextuelle nous a permis de mettre en évidence les enjeux et normes portés par les scientifiques : la peur de communiquer à d'autres scientifiques des éléments qui leur permettraient de publier, le temps limité dont les scientifiques disposent pour diffuser leurs connaissances, la multiplicité des canaux (outre les plateformes que nous leur proposions) pour échanger informations et connaissances.

L'approche systémique a permis de mettre en évidence les contextes dans lesquels ces scientifiques étaient immergés : des laboratoires évalués par des organismes tiers, une pression importante pour développer l'activité de publication, et des situations paradoxales dans lesquelles on demande par exemple à des scientifiques en concurrence de partager.

L'approche acteur-réseau a permis de mieux cerner l'évolution dans le temps (position et taille) des différentes composantes (acteurs et groupes d'acteurs). Enfin, plusieurs études sémiotiques (conduites sur les systèmes opérationnels) nous ont permis de comprendre - en complément des autres études - comment nous pourrions aménager les différents dispositifs.

De ces études SHS, nous avons extrait de nombreux enseignements : limiter les canaux de communication (pour valoriser la qualité de l'information mise à disposition), renforcer l'interactivité (donner des nouvelles à intervalles réguliers), privilégier le partage d'informations publiées (pour limiter les fuites de connaissances à haute valeur ajoutée), mettre en œuvre un déploiement progressif des différentes applications (pour soutenir l'intérêt des utilisateurs), réserver les applications d'échange direct comme les forums à des populations homogènes comme les doctorants (car les populations hétérogènes évitent de communiquer en mode non anonyme), etc.

À partir de ces constats, nous avons aménagé le dispositif et constaté une amélioration très sensible de son fonctionnement. Même si la fonction diffusion (de la direction des programmes vers les scientifiques) reste prédominante, le trafic à commencé à se développer (téléchargement des lettres d'informations, lecture des rapports d'activités et contributions des collègues, recherche de coordonnées, etc.).

Aujourd'hui nous ne conduisons plus les tâches STIC et SHS (étude et création de dispositifs) de façon séquentielle, mais simultanée (en particulier pour appréhender pratiques et usages qui sont difficiles à formaliser). Ceci permet de gagner beaucoup de temps dans la construction et la mise en œuvre de plateformes numériques de partage de connaissances, qui deviennent une composante importante des dispositifs destinés à améliorer la performance des collectifs. 


\section{Compatibilité et complémentarité entre modèles STIC et SHS}

Dans notre quête pour améliorer la performance des collectifs à travers la création de dispositifs instrumentés technologiquement, nous avons ensuite mis en œuvre des approches croisées : en constituant des équipes mixtes (STIC et SHS), en complétant la formation des spécialistes (des STIC vers les SHS, des SHS vers les STIC), et en élargissant le périmètre des modèles (en introduisant une dimension SHS dans des modèles STIC comme les diagrammes d'activités à couloirs temporels, ou en utilisant des modèles STIC pour documenter des modèles SHS à l'image des modèles de classes qui facilitent le repérage des acteurs clés à interviewer).

Voici un exemple d'élargissement SHS du périmètre d'un « modèle » STIC : celui du diagramme d'activités à couloirs temporels.

Le diagramme d'activités a vocation à matérialiser l'enchaînement des activités (généralement entendues dans le modèle STIC comme les tâches à accomplir par un processeur de calcul). L'idée, prévue dans le langage UML, est d'ajouter des « lignes de vie » pour chaque acteur ou groupe d'acteurs (qui représentent un calculateur ou des groupes de calculateurs en STIC) pour identifier plus facilement ce que va faire chaque « acteur » et la façon dont les traitements vont s'enchainer. Notre contribution est d'élargir le cadre d'étude en considérant que dans un grand nombre de dispositifs (semi-automatiques) les actions sont ou peuvent être confiées à des calculateurs comme à des individus. Ces derniers sont alors intégrés dans la représentation au même titre que les processeurs de calcul et bénéficient d'une ligne de vie. Le modèle est complété par certains aménagements méthodologiques : le modèle est construit via des entretiens qualitatifs non directifs centrés (individuels ou de groupe), aux besoins (généralement peu précis) sont préférées les exigences qui sont représentées par des rectangles pointant sur les activités. L'ensemble permet de co-construire avec les intéressés des modèles réalistes de la situation qui facilitent grandement la mise au point du dispositif.

Mais, les situations sont complexes et évoluent rapidement. De plus, les utilisateurs ont beaucoup de difficultés pour identifier et exprimer leurs «besoins ». Et dans ce cas, les études SHS même conduites dans les règles de l'art ne permettent pas toujours de collecter les bonnes informations.

Il y a toutefois quelques invariants qui émergent. S'il est difficile de prévoir ce qui va donner satisfaction, il est beaucoup plus facile d'identifier ce qui ne va pas fonctionner correctement. Le coût des investissements (en argent et / ou en temps) nécessaire pour créer un dispositif est important, et le coût de fonctionnement (pour aménager ce dispositif) généralement déterminant. Notre point de vue est que le temps est aussi une dimension trop souvent mal prise en compte dans les études (en SHS comme en STIC).

$\mathrm{Si}$ nous sommes aujourd'hui convaincu qu'il convient d'articuler modèles STIC et SHS, ces derniers doivent-ils être compatibles et / ou complémentaires? 
Notre point de vue est que l'approche doit être plus générale et le cadre plus large, en fait elle doit être centrée sur le dispositif (entendu ici du point de vue de Foucault). Les modèles STIC et SHS sont alors naturellement complémentaires, même s'ils ne sont pas directement compatibles. La compatibilité est dans ce cas assurée par le substrat du cadre général. Ce cadre général peut alors être appréhendé en différents espaces : espace de production, espace d'utilisation, espace des SHS et espace des STIC (Agostinelli, 2011). En effet, chacun de ces espaces est doté de sa propre logique, de sa propre dynamique.

\section{Pour un modèle d'efficience des collectifs}

Les travaux que nous avons conduits jusqu'à ce jour nous ont permis de progresser dans la compréhension du fonctionnement des collectifs pour chercher à en améliorer la performance. Toutefois, dans un contexte de concurrence élargie et de raréfaction des moyens cela ne semble pas suffisant. Il convient aujourd'hui de mettre en œuvre une autre étape dans la démarche : l'efficience des collectifs. L'efficience est ici entendue comme le rapport de la performance sur les moyens disponibles ou mobilisables.

Pour ce faire, nous proposons de débuter l'investigation - dans une approche interdisciplinaire - à travers quatre dimensions : la stratégie, les pratiques, les usages, et la régulation opérationnelle.

\section{Les pratiques}

Nous envisageons les pratiques comme la première composante d'un concept plus large qui est celui d'utilisation. En première approche les pratiques peuvent être décomposées en deux parties : la capacité à faire (ou compétences) et le souhait de faire (ou volonté et motivation).

Dès lors que nous nous intéressons à la question de l'efficience des collectifs dans un contexte de traitement semi-automatique (assisté par les TIC), la première question est de s'intéresser au potentiel des acteurs qui constituent ce collectif.

Bien entendu, il n'est pas question ici de réinventer une cartographie exhaustive des savoir-faire individuels et des ressorts de la motivation, mais de s'appuyer sur les travaux reconnus, comme ceux réalisés par exemple dans le domaine des référentiels métiers, pour construire un modèle des pratiques individuelles (compétences et motivations).

\section{Les usages}

Les usages constituent pour nous la seconde composante de l'utilisation. Dans notre acception les usages s'appuient sur l'ensemble des fonctions ou services mis à disposition des acteurs.

Ceci pose la question des tâches, le concept de tâche désignant ce qu'un individu doit ou peut faire (Leplat \& Hoc, 1983). La tâche peut être prescrite, 
c'est-à-dire imaginée et mise en place par l'autorité, ou effective, c'est-à-dire réellement effectuée par un individu dans le cadre de son activité.

Lidée est ici d'appréhender l'ensemble des fonctions ou services « utilisables » par les acteurs. Les tâches prescrites ou effectives peuvent alors être juxtaposées aux activités pour comprendre l'impact de la contribution d'un individu aux objectifs de l'entreprise ou de l'organisation.

\section{La stratégie}

Piloter une entreprise, une organisation, ou d'une façon plus générale une entité nécessite la mise en œuvre d'une stratégie et d'une régulation opérationnelle.

Dans des environnements de plus en plus complexes, composés en grandes parties de cadres dotés d'une assez large autonomie, la stratégie ne consiste plus à « programmer » l'ensemble des activités (une tâche = un opérateur), mais à ajuster l'ensemble des fonctions et services disponibles, et intervenir sur les pratiques.

\section{La régulation opérationnelle}

Arrêter une stratégie ne suffit pas, pour que le système soit efficace il convient de réguler son fonctionnement en temps réel. À l'image de l'entraîneur de football sur le banc de touche, il est indispensable de mettre en place un mécanisme de régulation souple et rapide, qui va permettre d'aménager le fonctionnement du système (de repositionner ou changer un joueur) dans l'intérêt du collectif.

\section{Conclusion}

L'idée est de construire un modèle d'efficience des collectifs en nous appuyant sur une démarche en compréhension et qualitative propre aux SHS. Ce modèle sera centré sur l'identification des compétences et des motivations (pour les pratiques), des fonctions et services (pour les usages), des objectifs et contraintes (pour la stratégie), des possibilités d'aménagements (pour la régulation opérationnelle).

Le modèle pourra alors être utilement complété à l'aide des concepts, méthodes et outils issus des STIC et plus particulièrement des travaux que nous conduisons depuis plusieurs années dans le domaine de l'aide à la décision dans le secteur industriel (Montmain, 2000)(Sanchez, 2007) (Imoussaten, 2011). Ces travaux sont centrés plus particulièrement sur : l'agrégation multicritère, les modèles de préférences basés sur la logique floue, et l'aide à l'arbitrage en situations complexes.

La combinaison originale des deux approches dans un modèle unifié du dispositif nous permettra alors d'élaborer des modèles de mise en œuvre et de pilotage, imaginés dans une perspective stratégique et déclinable dans une perspective opérationnelle. 


\section{BIBLIOGRAPHIE}

AGOSTINELLI S., La performance des technologies passe par la compatibilité des modèles SHS et STIC, Journées d'étude MTO'2011, Site de Nîmes de l'Ecole des mines d'Alès, 17 et 18 mars 2011.

AKRICH M., CALLON M. et LATOUR B., Sociologie de la traduction : textes fondateurs, Paris, Presses des Mines, 2006.

BOOCH G., RUMBAUGH J. and JACOBSON I., The Unified Modeling Language User Guide (2 ${ }^{\text {nd }}$ Edition), Addison-Wesley Professional, 2005.

FOUCAULT M., "Le jeu de Michel Foucault ", Dits et écrits, Tome III, Paris : Gallimard, 1977, p. 299.

IMOUSSATEN A., Modélisation et pilotage de la phase de délibération dans une décision collective : vers le management d'activités à risques, Thèse de doctorat préparée sous la direction du Pr. J. MONTMAIN, Ecole des mines de Paris, 2011.

JACOBSON I., CHRISTERSON M., JONSSON P. and OVERGAARD G., Object-Oriented Software Engineering : a Use Case Driven Approach, Addison-Wesley, 1992.

LEPLAT J. et HOC J.-M., "Tâche et activité dans l'analyse psychologique des situations ", Cahiers de psychologie cognitive, Vol. 3, n¹, 1983, p. 1389-1402.

MARTY C., MARTY R., 99 réponses sur la sémiotique, Edition du CRDP/CDDP Languedoc-Roussillon, 1992.

MONTMAIN J., Des modèles pour la supervision, Note de synthèse pour l'habilitation à diriger des recherches, Institut National Polytechnique de Grenoble, 2010.

MUCCHIELLI A., Théorie systémique des communications, Paris, Armand-Colin, 1999.

MUCCHIELLI A., La nouvelle communication, Paris, Armand-Colin, 2000.

PENALVA J.-M., Intelligence collective : rencontres 2006, Paris, Presses des Mines, 2006.

RICCIO P.-M., Une approche communicationnelle de la construction de projets innovants, Thèse de doctorat en sciences de l'information et de la communication préparée sous la direction du Pr. A. MUCCHIELLI, Université Montpellier III, 2003.

RICCIO P.-M., Automatisation cognitive : de la comprébension de situations complexes à la mise en cuvvre des technologies, Note de synthèse pour l'habilitation à diriger des recherches, Université d'Aix-Marseille, 2010.

SANCHEZ C., Spécification et implémentation d'un modèle d'aide à la décision multicritère pour la maintenance préventive et la gestion du patrimoine de la société d'autoroute ESCOTA : le projet SINERGIE, Thèse de doctorat préparée sous la direction du Pr. J. MONTMAIN, Ecole des mines de Paris, 2007.

STEFIK M., BOBROW D., «Themes and Variations ", AI Magazine, Volume 6, Issue 4, winter 1986, p. 40-62.

Résumé : L'objectif de cet article est de jeter les premières bases d'un modèle d'efficience des collectifs pour améliorer de façon progressive la performance d'un collectif dans l'action en relation avec les moyens qui sont disponibles ou peuvent être mobilisés. Nous 
proposons une approche fondée sur les sciences de l'information et de la communication articulée autour de la notion de dispositifs, formels et informels, pour comprendre la situation. Notre idée consiste ensuite à juxtaposer pratiques (compétences et motivations) et usages (fonctions et services) pour conduire une régulation opérationnelle à court terme et imaginer puis mettre en œuvre une stratégie à plus long terme.

Mots-clés : Approches qualitatives, modèles de connaissances et d'information, pratiques, usages, sciences de l'information et de la communication.

Abstract : The objective of this paper is to lay the foundations of a model of collective efficiency to improve progressively the performance of a community action in relation to the means that are available or can be mobilized. We propose an approach based on information and communication science organized around the notion of devices, formal and informal ones, to understand the situation. Our idea is then to juxtapose practices (skills and motivation) and uses (functions and services) to conduct a regulatory short-term operational and also imagine and then implement a strategy in the longer term.

Keywords : Qualitative approaches, Knowledge and information modeling, Practices, Uses, Information and communication sciences. 